\title{
African Rhythmic Identity and Creativity Art in Pepeye Owubantutu: A Yoruba Folksong Arrangement By Debo Akinwumi
}

\begin{abstract}
David Bolaji, $P h D$
Department of Music, University of Port Harcourt, Choba, East- West Road,

Abstract.

The creativity and uniqueness of modern African art composers in using the indigenous musical materials stand as media for revealing the African rhythmic identity, artistic ingenuity, cultural features, and the schools of thought of the composer(s). The distinctive expression continuum of a modern African art composer presents the skilful arts and intellectual ability of the composer, via his/her work(s). In view of this background, the Yoruba folksong arrangement of Pepeye Owubantutu for voices, (Soprano, Alto, Tenor, Bass) by Debo Akinwumi, reveals the uniqueness of African rhythmic identity found in the Yoruba milieu. Empirical research method was used by the researcher to justify the thrust and findings of this study. The study serves as educative resource material to the young modern art composers. It exposes diverse compositional techniques which can be used in adopting and applying the indigenous folksong into modern art music. Furthermore, it suggests strategies for building art composers to adopt and promote African rhythm in their art compositional works.
\end{abstract}

Keywords: Folksongs, Creativity, Indigenous and African rhythm identity.

DOI: $10.7176 /$ RHSS/9-6-08

Publication date:March $31^{\text {st }} 2019$

\section{Introduction}

The introspective aim of every modern African art composer that uses indigenous materials/folksongs in art compositional style is not to propagate the acculturation of Western and African idioms alone. Rather, its premeditative objective might centre on the indicative of his/her cultural musical heritage and the textural rhythmic identity of his/her culture, as it reflects and represents the African rhythmic identity. Similarly, the propagation of his/her musical skilfulness may not be ruled out, as well as the artistic compositional techniques within the circumference of art music composition. In achieving this, structuring/arranging African folksong(s) in the Western art musical style stands as a strong medium that composers had used in their compositions in Nigeria and globally. Although, the textural rhythm, melodic phraseology and inflective text prose as captured in the indigenous language of a folksong, as well as other musical conglomeritic components serve as the basic elements/ingredients for presenting part of the cultural norms and the African identity of a particular milieu. (Nzewi 2017). According to Samuel (2013),

Traditional ethnic music, notably folksongs, as a culture indicator provides an avenue for clear expression to the level of cultural complexity, and a set of norms differentiating and sharply characterizing cultures when analysis of their song texts are systematically embarked upon.

In the same vein, Onyeji (2016) affirms that, music in Africa serves as an inevitable form that presents and sustains the socio-cultural norms and values that characterise the coexistence of a particular culture. Furthermore, he identifies folksong as a notable genre, among others that constitutes the phenomenon of music in Africa. In his view, the continuous tutelage of folksongs through the oral procedure from one generation to the other stands as the laid down institutional structure in Africa for educating her society on moral and sociocultural issues.

Nonetheless, the arrangement of folksong in art compositional style can be seen as a paradigm for announcing and preserving diverse indigenous norms and attributes of various African cultures across the globe. Viewing the above submission through the atomic paradigm in the scholastic domain, the skilfulness and experiences of an individual art composer justify the intellectual gamut that can be unravelled from any of his/her folksong arrangement. Using a microscopic gamut to dissect and analyse the content of the composition under study, one could conclude that the folksong arrangement of Pepeye Owubantutu by Debo Akinwumi affirms and reflects the embodiments of Africanism; meaning that, the encapsulation of African rhythmic identity and other musical elements via the application of Akinwumi's creativity in his folksong arrangement, characterizes and presents the indigenous musical attributes of the Western Nigeria (Yoruba), as displayed through his compositional germs. Samuel (2013), buttresses that some of the cultural values and expressions of the Yoruba can be found in their folksongs. This he affirmed within the empirical study of the Egbeda-Egga women's folksongs. This 
particular attribute is as well found in the Sub-Saharan region of Africa in the Southern Nigeria among the Igbo. (Nzewi 1991 and Onyeji, 2016.).

\section{African Rhythmic Identity}

Speaking and viewing the rhythmic identity in Africa, it is highly expedient at this juncture to reinstate the idealistic perception and opinion of the word "rhythm" and "African rhythm" as stated by some scholars. The reaffirmation and the definition of rhythm given by New Harvard dictionary and Oxford dictionary were clearly reinstated by Vidal (2003), to be "everything pertaining to the temporal quality or duration of musical sound and regular succession of weak and strong stresses, accents sound or movements in speech, music and dancing". He further buttressed that, rhythm is a pulse in space that is arranged systematically through time. Similarly, Atinuke (2002) account of rhythm re-established the working definition given by Webster (1990) and Crystal (1992), that stated rhythm to be the "forward movement of music'. This simply means the formation and subdivision of shifting accents in relation to the recurrence of a particular pattern and the steady flow of beats in a given time, said to be rhythm. Looking at rhythm from a different perspective, it could be taking as the holistic sense of musical progression having the steady and the non steady pulse embedded in it. (Apel, 1973 and Atinuke, 2002). In the same vein, rhythm stands as the fundamental phenomenon that made up of sonic and silence that binds the musical elements together within a steady flow pattern. That is to say, the recurrence of strong and weak beat with a regular and irregular flow of sonic, form the basis of rhythm in a holistic overview of any music. Ofuani (2014) states that, rhythm serves as the basic phenomena that integrate other elements that constitute the holistic features of its existence together. However, the concept, structure and functionality of the rhythm differ in nature from one continent to another.

The notion of African rhythm using the micro and macro dichotomy to justify its exceptionality among other rhythms of the world, distinguishes its stands when compared with others. This has paved the way for more introspective investigations and insight researches on African rhythm than any other rhythm of the world. In the view of Kauffman (1980), African rhythm is the rhythmic pattern that has captured the interest of numerous ethnomusicologists. Furthermore, he buttressed the fact that much has been presented in a universal view to explicate the phenomenon of African rhythm. Agawu, (1987) upheld the ethno-musicological view that projects African rhythm as a complexity and culturally rhythm that reveals the holistic entity of the Africans. Similarly, Seeger, (1966) acknowledges that African rhythm is an integrated rhythmic pattern that is played and absorbed in a unified entity without any perceive of isolation. This means that, the concept of a principal melody and subsidiary harmonic parts which could be identified in the Western music style does not apply and represent the ontology of African rhythm. Nzewi (2007) defined African rhythm as the basic fundamental of different pulses that reflects the flow of an energetic African musical beat. He argues that the multifaceted sound that comes through the interface of diverse pulse movement aligned together to produce a unique singular rhythm. It is on this platform that this study seek to identify some of the peculiar rhythmic patterns and their features as used and applied in the Yoruba folksong arrangement of "Pepe ye owubantutu."

The ideological view of African rhythm and its identity stand out in a unique form in all of the African cultures and beyond. This is due to the distinctive nature, features and musical elements that are clearly found and embedded in it. The simulations of this rhythm has been investigated into and classified to be fascinating. Which mean, African rhythm is a fascinating rhythm that plays a potent functional role in the context of African musical performance. Speaking on the fascinating gamut of African rhythm, scholars have affirmed, and reaffirmed, that the rhythmic components of constituting the coherent simulation of African rhythm have a potent integral relationship that connects Africans, music and society together. By implication, African rhythm does not hinged its predominate role on the premise of just being an accompaniment in a performance, as is found within the context of the Western music performances.

The uniqueness of African rhythm has been seen, captured and stated as melo-rhythmic in nature. This in line with other conceptual backgrounds that has describes African rhythm as a cross rhythm, complex rhythm and poly-rhythmic patterns. etc. (Nzewi, 1974; Agawu, 1987; Arohm 1973. and A. M Jones, 1952.). However, using Nigeria as a reference point from the Sub-Saharan region of West Africa, Nigeria as a single entity has witnessed strong influences on its musical and religious landmarks and socio-cultural practices at the advent of colonization. Omojola (1997) It is of no doubt that all of these landmarks has influenced most musical genre in Nigeria. These influences have resolve in the introduction of new musical idioms, alongside with the practices of Christian and Islamic religions which were totally different from the indigenous religion practices in Nigeria. Nevertheless, the generic influence that was experienced as the result of colonization in Nigeria has not overshadowed and changed completely the totality of the indigenous music of the Yoruba's in Nigeria. Rather, the generic root of African rhythm still stands within the contextual music and performance practices of the 
Yoruba; although, the positive impacts of Western art music and its applications now serve as paradigms through which the indigenous music are being sustained and documented. The re-arrangement of Nigeria folksongs in an art form tends towards repositioning and presenting it stance globally.

\section{African Rhythmic Identity in Akinwumi’s Compositional Techniques}

The ingenuity of Akinwumi's compositional style and identity hinged on his creative ability in using the indigenous musical elements of his culture (Yoruba folksong), as the fundamental bedrock in promoting African rhythmic identity. The presentation of African rhythmic identity through the compositional techniques as used by the composer in the folksong arrangement of Pepe ye Owubantutu, can be seen and interprets according to the skilful act of the composer. This African rhythmic identity is identified and grouped into four different rhythmic fragments in this study. These include: pre-model rhythmic harmonic rule of principal voice, recurrence rhythmic pattern, subjective rhythmic pattern and the complementary rhythmic pattern. The first underscore phraseology of this composition started on the second half beats which eventually established anacrusis beat in the music. The rhythmic structure of this first phrase started with an anacrusis beat to the fourth (4th) measure. This serves as a repetitive rhythmic structure that was recaptured in measures twelve to sixteen, $(12-16)$ in the folksong arrangement. The frequency level in recapturing the same rhythmic patterns was established in direct imitative patterns that occur in a major second above the first note (B-flat note). This was seen and taken by the tenor and bass parts respectively. However, the functionality of this rhythmic pattern serves as the time-line that gives and dictates the pace and flow of the entire music and it stands as the main motif of the music as well. This repetitive rhythmic pattern is referred to as recurrence rhythmic identity in this study. This specifically symbolizes and represents the attributes of African rhythmic identity as it found in most African indigenous ensemble performance. The rhythmic principle/motif symbolizes the fundamental role that could be ascribed majorly to any African indigenous musical instruments that plays the role of the timer within the context of an indigenous ensemble performance. According to Nketia (1963)

One of the regular means of establishing and maintaining the beat is the accompanying rhythm played by the idiophone. Any of these [gong, woodblock etc.] provides a timeline, a constant point of reference by which the phrase structures of a song as well as the linear metrical organization of phrases are guided.

In line with the above, it is an established submission that the indigenous instruments such as agogo, (metal-bell) and wood block in West Africa are saddled with the responsibility of time-line rhythm (timer).

\section{PEPEYE OWUBANTUTU}

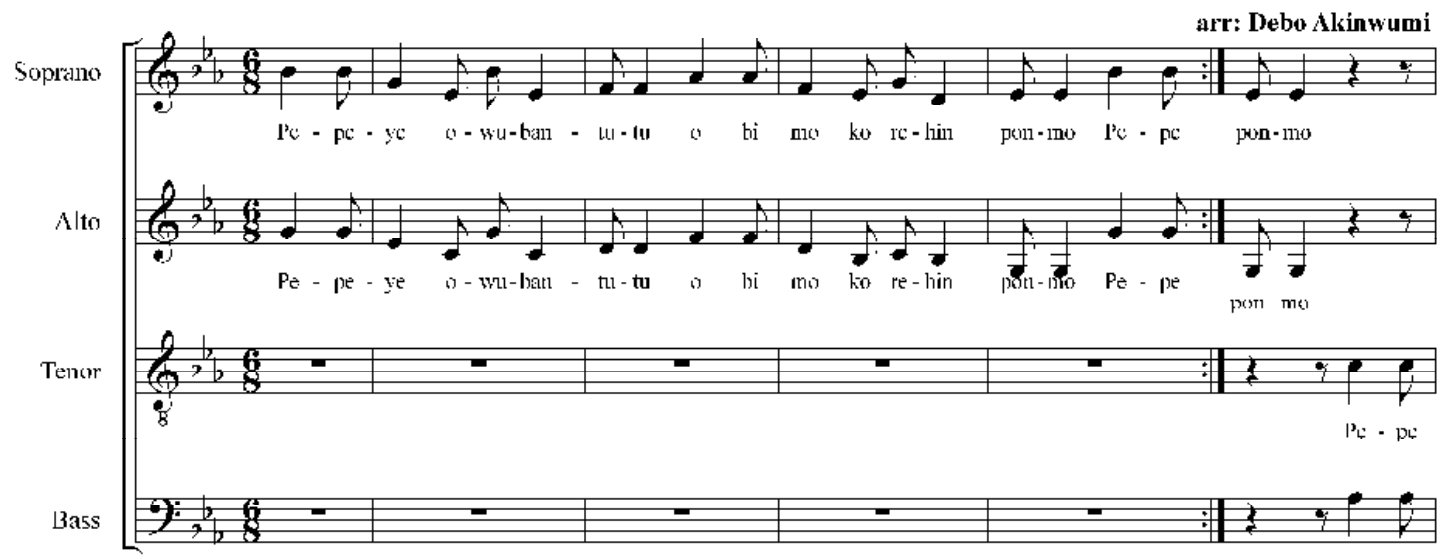

lïg..1 recurrence ryhthmic identity

In the same vein, modern Nigerian art composers have used fragmentation/excerpt from indigenous musical materials as a major motif/subject that compositional works are built on. This singular act affirmed the dual functionality of the recurrence rhythmic identity as its plays the role of time-line, as well as the main motif of 
the music. This can be seen within the context of the understudy composition from the anacrusis beat to measures sixteenth. Onyeji (2008), adopted similar derivative rhythmic pattern from the indigenous musical materials of the igbo, which he used to conceptualized his drummistic-piano techniques. He emphasises that the single rhythmic entity that recurred in a particular musical performance could serve as the platform of expressing the creative vocabularies of a composer. Furthermore, in his explicit explanation of his creative work, he termed such rhythmic pattern as melo-rhythmic pattern that stands as the backbone/source of his creativity. Basically, recurrence rhythmic identity in this study serves as one of the major rhythmic identities that characterizes and distinguishes African rhythm from other rhythms of the world in any musical performance.

Viewing the harmonic context of the composition under study, it is obvious that the composer employs the Western harmonic style. The contextual harmonic rhythm in the study was used to create and achieve the harmonic texture of the study. Specifically, its exposes and serves as the paradigm that explains the applicable technique(s) that the composer uses in achieving what I referred to as pre-model rhythmic harmonic rule of principal voice. This occurs in the following measures, the anacrusis measure -16 and $26-47$, which involves alto, tenor and bass parts); (section D), measures 60 - 67 and; (section H) into (section I), measures 102 - 130. In this context, the composer reduplicates the exact rhythmic structure of the leading voice (Soprano) to the subsidiary parts, (alto, tenor and bass) in conformity with the rules and regulations that govern the Western harmony, which means that the, rhythmic structure of the principle/leading voice (Soprano) stands as the premodel rhythmic structure that other parts were patterned after. Furthermore, the total harmonic proceeding from the beginning of the music (anacrusis beat), to measures 16 into 17 presents vertical rhythmic harmonic structure. The introduction of subjective rhythmic structure in all the voice parts starting from measures eighteen to twenty-three $(18-23)$ gives the harmonic structure a different perspective. The harmonic rhythmic procedure in measure eighteen (18) enables the establishment of what I referred to as complementary rhythmic movement. In this context, each rhythmic structure of the voice part contributes to the over-all entity of the harmonic structure of the section. Also, each voice part plays a complementary rhythmic role together with the common aim and goal of achieving a single harmonic entity. This particular view characterizes the uniqueness of African rhythmic identity within the context of an indigenous ensemble performance. It explains the pre-modal aim and objective of different rhythmic structures that coexist through connective links of having a common goal of achieving a single rhythmic entity in African musical performance. The place of isolated rhythmic structure/movement of a particular part does not occur within the circumference of African rhythmic identity. Rather, each rhythmic structure serves as a unifying phenomenon within the performance context in African milieu. Although, the composer uses the pre-modal rhythmic and complementary rhythmic pattern within this measures. Measures $21-22$ represent the combinations of the two rhythmic patterns. The pre-modal rhythmic structure was used between the soprano, alto and the tenor parts, while the bass part adopts a complementary rhythmic structure, conforming to the structural movement of other parts.

The beginning of measure 24 established the section B of this work. This section combines the integral use of pre-model rhythmic harmonic rule of principal voice that brought in the repetitiveness of the same rhythm in an interval of an octave lower. This established and gives the identifiable technique of call and response as used by the composer. Likewise, recurrence of rhythmic idea as stated in section B between measures $24-48$ were recaptured in section $\mathrm{C}$ in measures $49-56$. The composer uses an antigenic rhythmic mode to create a strong contrast in measures $137-154$. This was taken by soprano as solo section, and followed by a duet section for Alto and Tenor between measures 155 - 178; adopting a pre-modal rhythmic movement.

The composer uses two of the rhythmic patterns identified in this study to make the final statement of his composition. These rhythmic patterns are pre-model rhythmic harmonic rule of principal voice and complementary rhythmic movement. These two rhythmic structures occur between measures $183-196$. The pre-modal rhythmic harmonic rule of principal voice was adopted between three voice parts; soprano, alto and tenor parts, while, the complementary rhythmic movement was used for the bass part in providing and giving harmonic proceeding that corresponds to the harmonic formation of each chords as used and applied by the composer.

\section{Conclusion}

In an attempt to justify the thrust of this study, four different rhythmic structures were identified within the circumference of the composition under study. These include: pre-model rhythmic harmonic rule of principal voice, complementary rhythmic movement, recurrence rhythmic movement and subjective rhythmic pattern. These identified rhythmic patterns were revealed and noted as strong rhythmic patterns that are peculiar and 
rooted within the African music. Furthermore, these rhythms serve as phenomena that showcase the African rhythmic identity as used by the composer in this work.

Basically, the concept of rhythm serves as the bedrock of creativity in any art compositional work in Africa. Similar, rhythm within the African milieu stands as the basic nature of the African music and its performance, as well as a potent phenomenon that integrates the norms, culture and the believe system of a particular society. The principal factor in African rhythmic identity is its fascinating structure. This has given different modern art composers opportunity to explore, interpret, propagate, and sustain the proclamation of African rhythmic identity through individual arts musical skills.

An abstractive over-view of the Composition Pepeye Owunbantutu, composed by Debo Akinwumi was looked into, in order to explore some of the proceeding techniques used in presenting African rhythmic identity through his compositional vocabularies. This study advocates that modern African art composers need to adopt and use more of indigenous materials in art musical compositional style. This will serve as an antidote to the extinction impending that most of the folksongs scripted and documented procedure(s) of preserving folksongs in African; and the potent digitalization and civilization erosion that is sweeping away the fundamentals nature of African rhythm.

\section{References}

Agawu, V. Kofi (1987). The Rhythmic Structure of West African Music: The Journal of Musicology, Vol. 5, No. 3 (Summer, 1987), pp. 400-418 Published by: University of California Press Stable URL: http://www.jstor.org/stable/763699 Accessed: 07-09-2016 02:42 UTC

(1995). African rhythm: A northern Ewe perspective, New York: Cambridge University Press.

Arom, S. (1973). African Polyphony and Polyrhythm: Musical Structure and Methodology

Atinuke. A. A. (2002). "Rhythm: A Dimension of Understanding African Creative Drumming”. In E. O Idolor (ed). Music in Africa. Facts and Illusions. Ibadan: Stirling-Horden Publisher (Nig.) Ltd. 73 - 81

Jones, A. M. (1952) “African Rhythm”, African Music No. 24 pp 26-47

Kayode, M. Samuel. (2013). African Folksongs as Veritable Resource Materials for Revitalizing Music Education in Nigerian Schools Research on Humanities and Social Sciences www.iiste.org ISSN 2222-1719 (Paper) ISSN 2222-2863 (Online) Vol.3, No.10, 2013

Nketia, J.H.K. (1963). African Music in Ghana. Evanston: North-western University Press. Nzewi, M. (1974). A Contemporary Study of Musical Arts: Informed by African Indigenous Knowledge Systems. vol. 1. vol. 2. vol. 3. vol. 4. vol. 5

, (1991). Musical Practice and Creativity - An African Traditional Perspective. Bayreuth:IwalewaHaus, University of 'Bayreuth

(2007) A Contemporary Study of Musical Arts: Informed by African Indigenous Knowledge Systems. Pretoria, South Africa: CIIMDA (Centre for Indigenous Instrumental Music and Dance Practices of Africa), 2007. 5 volumes (vol. 5 includes 3 books of music scores) +2 volumes of educational materials, CD included. ISBN vol. 1: 97819200516

(2017) Reinstating The Soft Science of African Indigenous Musical Arts for Humanity sensed Contemporary Education and Practice

Ofuani, S. (2014). Traditional Rhythmic Patterns: The Source of Creativity and Identity of Original Nigerian Art Music Compositions. International Journal of Humanities and Social Science: Vol. 4, No. 9(1); July 2014

Omojola, B. (1997). Compositional style and national identity. In Omojola (Ed.), Music and Social Dynamics in Nigeria, (pp. 209-219). Ilorin: Department of Performing Arts. 
Onyeji, C. (2008). Drummistic Piano Composition: An Approach to the Teaching of Piano Composition from a Nigerian Cultural Perspective. International Journal of Music Education (IJME), No. 26 vol 1. Pp 161175

(2016). Composing Art Music Based on African Indigenous Musical Paradigms An inaugural Lecture Summary presented on 11, 2016, at the University of Nigeria Nsuka.

Seeger, C. (1966). "Yoruba Dundun Drumming for the Hunters (Iiuode), A Discourse of its Text" . Journal of Arts and Ideas. Obafemi Awolowo University, Ile-Ife Pp. 79-92

Vidal. T. (2003). Rhythm and Metre in Yoruba Songs, Nigeria Music Review Vol. 4 2003: 1 -16

https://www.merriam-webster.com/dictionary/rhythm http://www.davidcrystal.com/?id=3117

\section{The Compositions under Reference}

Onyeji, C. (2008). Nka Emume. South Africa: Platinum Press.

Debo, A. (2008). Pepeye Owubantutu. Nigeria: Most Excellency Music. 